Ann. Zootech., I976, 25 (4), 52I-53I.

\title{
QUANTITÉS D'HERBE ET DE LAIT CONSOMMÉES PAR DES VEAUX AU PIS
}

\author{
P. LE NEINDRE*, M. PIETIT* et A. MULLER** \\ avec la collaboration technique de G. PEcatte et Y. Gallard \\ * Laboratoire de la Production de Viande, \\ Centre de Recherches de Clermont Ferrand, I. N. R. A., \\ Theix, Saint Genès Champanelle, 63110 Beaumont \\ ** Domaine expérimental du Pin, I. N. R. A., \\ (Productions animales), Le Pin au Haras, \\ 61310 Exmes \\ RÉSUMÉ
}

Au cours de deux années, on a mesuré les quantités de lait et d'herbe consommées entre l'âge de 3 et 8 mois par 96 veaux, Normands ou croisés Charolais-Normands, élevés au pis à raison de 3 veaux par vache. Une augmentation de la consommation de $\mathbf{~} \mathrm{kg}$ de lait a entraîné une augmentation significative de $65 \mathrm{~g}$ du gain de poids journalier, aussi bien entre 3 et 5,5 mois qu'entre 5,5 et 8 mois.

La consommation d'herbe augmente considérablement entre 3 et 8 mois, de I, I 2 à $4,76 \mathrm{~kg}$ de matière sèche par animal et par jour. Par kilogramme de poids métabolique, les veaux ont ingéré d'autant plus d'herbe qu'ils consommaient moins de lait ; cependant, en énergie métabolisable, la compensation d'une diminution de la quantité de lait bue par un accroissement de la quantité d'herbe consommée n'a été que de 44,3 p. Ioo entre 3 et 5,5 mois, et de I I,6 p. Ioo entre 5,5 et 8 mois.

Les quantités d'énergie métabolisable disponibles pour la production d'un kilogramme de gain ont été identiques quelles que soient les proportions respectives du lait et de l'herbe ingérés, et ont augmenté avec l'âge de 4233 à $5876 \mathrm{kcal}$.

\section{INTRODUCTION}

Le veau traditionnellement élevé avec sa mère au pâturage jusqu'à l'âge de 7-8 mois consomme essentiellement du lait et de l'herbe. Pour améliorer la croissance de ce veau sous la mère, il importe de mieux connaitre l'influence sur cette croissance 
des quantités respectives de ces aliments et leurs interrelations. La plupart des auteurs ont cherché à définir la liaison existant entre la croissance du veau et la quantité de lait bue (Brumby et al., I963; Merton et al., I967; Furr et Nerson, I964; PoujARDIEU, I969, etc.). Ils ont noté que l'intensité de la liaison et aussi la pente de la droite de régression diminuaient avec 1'âge du veau. Ils ont supposé que cela était dû̀ à la diminution de l'importance du lait dans l'alimentation du veau sans toutefois mesurer les quantités des autres aliments qui étaient consommées.

Dans la présente étude, nous avons mesuré l'évolution des quantités d'herbe ingérées par des veaux élevés au pis entre les âges de 3 et 8 mois. Ces veaux recevaient des quantités de lait différentes selon le potentiel laitier de leur nourrice. La production laitière de ces vaches a été analysée dans une publication antérieure (LE NEINDRE: ct al., I976 a). Nous chercherons à préciser à partir cle ces données l'influence respective du lait et de l'herbe sur la croissance cles reaux.

\section{MATÉRIEL ET MÉTHODES}

En 1972 et 1973. I6 vaches Normandes, avant vêlé en moyenne le 19 et le 26 lévrier respectivement pour la première et la seconde année, ont allaité chacune simultanément leur propre veau (Normand ou Normand-Charolais) et cleux veaux supplémentaires achetés (croisés Normand $\times$ Charolais la première année, et Normands la seconde année). L'origine et le mode de conduite des vaches ont été décrits dans une étude antérieure. Les deux veaux supplémentaires étaient affectés à chaque vache le plus tôt possible après le vêlage (deux jours en moyenne) ; ils étaient âgés de I à 2 semaines la première année et de 2 à 5 semaines la scconde année. Au total 85 veaux mâles et Ix veaux femelles ont été allaités.

Les veaux sont restés constamment en stabulation libre. Ils tétaient les vaches deux fois par jour sous surveillance à 8 heures et à I 8 heures. Jusqu'au 6 mai en I972 et au r 4 mai en I973, ils avaient à leur disposition du foin et un aliment concentré à volonté. Ils ont ensuite reçu jusqu'au sevrage (le 18 octobre les deux années) du ray-grass anglais fauché une fois par jour et distribué en deux repas à volonté ( 12 p. Ioo de refus).

Chaque année, avant le début de la clistribution d'herbe aux veaux, on a réparti les vaches en 4 lots qui différaient par la quantité de lait produite: un lot de fortes productrices (lot $\mathrm{H}: 7,99 \mathrm{~kg}$ et $6,85 \mathrm{~kg}$ (e lait bu par jour et par veau pendant la période expérimentale, respectivement en I972 et I973), 2 lots de moyennes productrices (lot M: 6,62 kg et $6,25 \mathrm{~kg}$ en I972;6,52 kg et $5,87 \mathrm{~kg}$ en $\mathrm{I973}$ ) et un lot de faibles productrices (lot B :6,02 $\mathrm{kg}$ et $5,40 \mathrm{~kg}$ ). I.cs douze veaux d'un même lot de vaches étaient affourragés ensemble dans une même case. Dans la présentation des résultats, les 2 lots intermédiaires dont les vaches avaient des productions comparables, ont été regroupés.

Du i9 mai au 28 septembre la prenière année, et du 23 mai au 9 octobre la seconde annéc. on a mesuré cinq jours par semaine les quantités d'herbe distribuées et leurs teneurs en matière sèche à chaque repas, ainsi que les quantités d'hcrbe refusécs ct leurs teneurs cn matière sèche une fois par jour. L'âge moyen des veaux était de 95 jours au début et de 236 jours à la fin de la période cle mesures.

Simultanément, la digestibilité de l'herbe a été mesuréc gràce à 3 béliers en cage à bilan qui ont consommé la même herbe que les veaux, pendant dix semaines en 1972 (du $26-6$ au 3-9) et pendant toute la période expérimentale en I973 (vingt semaines). Lorsqu'il n'y avait pas de mesure de la digestibilité en I972, nous avons utilisé les valeurs présentées par DemarquiLLy et WeIsS ( 1970 ) dans leurs tables dont une partie des données provient du même domaine (fig. I).

Dans l'analyse des résultats concernant le gain de poids vif des veaux et la quantité de lait bue, on a utilisé la moyenne des 3 veaux au pis d'une même vache, et dans celle des résultats concernant les quantités d'herbe consommées, la moyenne des I 2 veaux d'un même lot.

Cette étude a été limitée à la période pendant laquelle les quantités d'herbe ingérées par les veaux ont été mesurées ( $1+3$ jours en 1972 et 1 4o jours en 1973 ). L'examen des quantités d'herbe consommées par les veaux nous a conduit à diviser cette période en deux parties ayant approximativement la même durce (environ $7^{\circ}$ jours). 


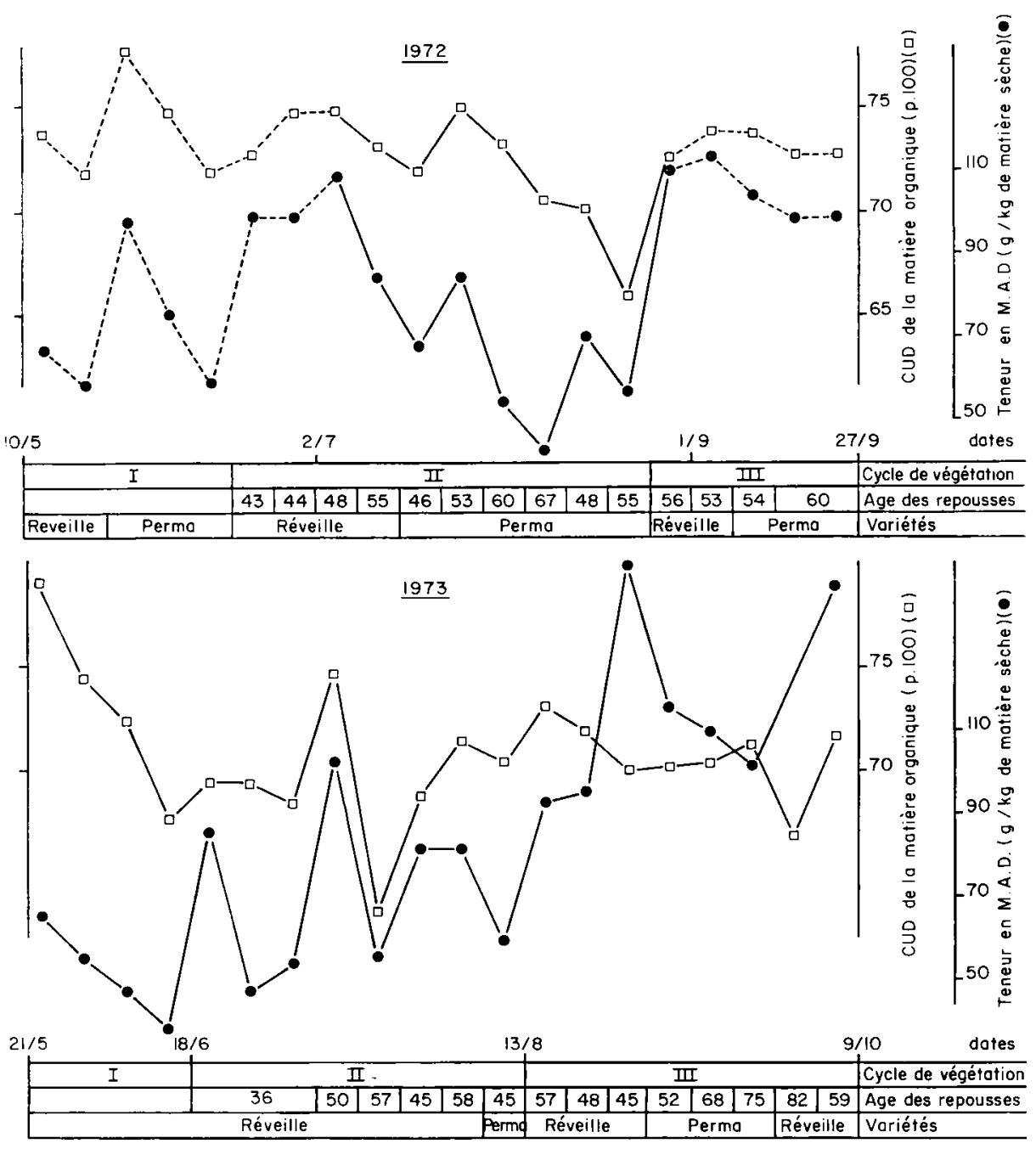

Fig. $x$. - Fivolution du coefficient d'utilisation digestive (C.U.D.) de la matière organique et de la teneur en matières azotées digestibles (M.A.D.) du ray-grass anglais consommé par les veaux

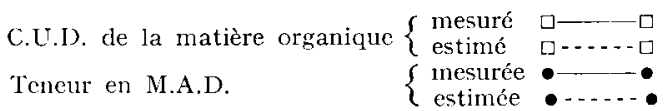

\section{RÉSUITATS ET DISCUSSION}

I. - Crô̂t des veaux et influence de la quantité de lait bue

Le poids moyen des 96 veaux a augmenté du $95^{\circ}$ au $236^{\circ}$ jour de II $5,4 \mathrm{~kg}( \pm 24,4)$ à $27 \mathrm{I}, 3 \mathrm{~kg}( \pm 32,4 \mathrm{~kg})$, soit une croissance journalière de I Io6 g, constante pendant pratiquement toute la période expérimentale (fig. $2 c$ ). La croissance des I I femelles 


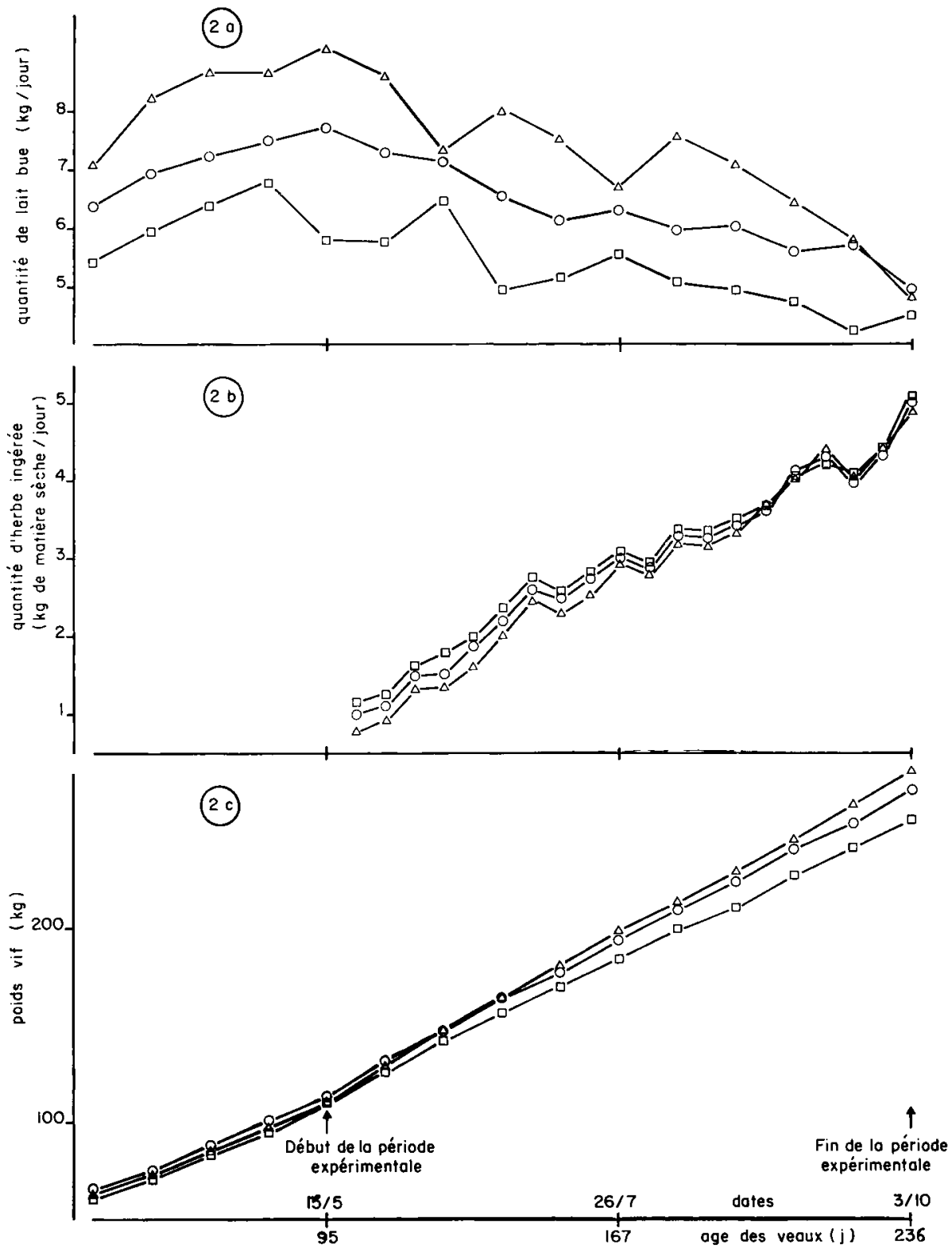

FIG. 2. - Évolution des quantités de lait bues (2 a), des quantités d'herbe ingérées (2 b) et du poids vif $(2 c)$ des veaux recevant des quantités de lait différentes

$$
\text { Quantité de lait bue } \begin{cases}\text { Importante } & \Delta \\ \text { Moyenne } & 0 \\ \text { Faible } & \square\end{cases}
$$


( I I $20 \mathrm{~g} / \mathrm{j}$ ) a été semblable à celle des I9 veaux mâles élevés sous les mêmes vaches ( $\mathrm{I}$ O50 $\mathrm{g} / \mathrm{j}$ ).

Les quantités de lait consommées ont varié selon les veaux de 9,26 kg à 4,69 kg pendant la première période et de $8,23 \mathrm{~kg}$ à $4,5^{\circ} \mathrm{kg}$ pendant la deuxième période. Le gain de poids vif des reaux a varié dans le même sens que la quantité de lait bue. Une analyse de covariance tenant compte de l'année a montré que les corrélations intra-années entre la quantité de lait bue et le gain de poids sont identiques pour les deux périodes successives $(0,67$ et 0,65$)$. Les augmentations de croît par kilogramme de lait supplémentaire estimées par les coefficients de régression intra-années sont également identiques entre périodes (66 et $64 \mathrm{~g}$ ). Ce résultat est sensiblement différent de ceux observés sur des veaux allaités par leur mère au pâturage (NEVILLE, I962; BRUMiry et al., I963; LE NEINDRE et al., r976 b), pour lesquels ces deux paramètres diminuent lorsque l'âge augmente. Cela peut s'expliquer par le fait que la variabilité des croissances, souvent importante pour les veaux âgés au pâturage est plus réduite dans cette étude ; en particulier, chaque résultat correspond à la moyenne de 3 veaux allaités par une même vache et les conditions de milieu sont plus homogènes qu'au pâturage (veaux à l'intérieur, séparés de leur nourrice, et recevant la même herbe coupée).

L'utilisation des axes principaux au lieu des droites de régression permet de mieux exprimer la liaison fonctionnelle pouvant exister entre le poids et la quantité de lait bue (Poujardieu, I969). Ainsi calculée, l'augmentation du gain de poids par kilogramme de lait supplémentaire est plus élevée et voisine de $98 \mathrm{~g}$ que ce soit de 3 à 5,5 ou de 5,5 à 8 mois. Ce résultat est très voisin de ceux observés par PoujARDIEU (I969) ou par nous-mêmes dans des populations de race Limousine (83 et roo g respectivement de o à 3 et de 3 à 6 mois) et Charolaise et $A$ ubrac (Ioo à $87 \mathrm{~g}$ ). Finalement, il semble que l'effet des variations de quantité de lait bue reste toujours aussi important quel que soit l'âge des veaux, malgré la diminution relative de l'importance du lait dans la ration totale.

\section{II. - Quantités d'herbe consommées}

Les quantités d'herbe consommées par les veaux ont augmenté considérablement du $95^{\mathrm{e}}$ au $23^{\mathrm{e}}$ jour (fig. 2 b). Elles sont passées de I, I2 à $4,76 \mathrm{~kg}$ de matière sèche par jour et par animal, soit de $0,9 \mathrm{I}$ à $\mathrm{I}, 76 \mathrm{~kg}$ de matière sèche par jour et par Ioo $\mathrm{kg}$ de poids vif et de 50 à $7 \mathrm{r} \mathrm{g}$ de matière sèche par jour et par kilogramme de poids métabolique $\left(\mathrm{P}^{0,75}\right)$, Cet accroissement des quantités d'herbe ingérées avec l'âge est plus réduit que celui olsservé par ALDER (I970) sur des veaux Hereford $\times$ Frison sevrés à l'âge de 2 à 3 mois. Mais il montre cependant l'importance croissante que peut avoir la quantité et la qualité de l'herbe disponible sur la croissance des veaux, particulièrement au-delà de 4 à 5 mois.

Les quantités d'herbe et de lait ingérées par kilogramme de poids métabolique varient en sens inverse, aussi bien pendant la première période $(\mathrm{P}<0,025)$ que dans la seconde $(\mathrm{P}<\mathrm{O}, \mathrm{OI})$. Ces différences d'appétit sont à rapprocher de celles observées durant la phase lactée par MATHIEU et LITRE (I96I) sur des veaux recevant différentes quantités de lait et un aliment concentré à volonté ou par GLEESON (I97I) sur des veaux à l'herbe. Elles semblent liées au développement relatif plus rapide 
des compartiments digestifs, notamment $d \mathfrak{u}$ rumen, chez les veaux recevant moins de lait (Mathieu, I96I; Kaiser, I973).

En revanche, c'est uniquement at1 cours de la première période que la quantité de matière sèche totale ingérée par animal diminue significativement $(\mathrm{P}<0,025)$ lorsque la quantité de lait bue augmente $\left(-3^{6} \mathrm{~g}\right.$ d'herbe par kg de lait supplémentaire). Des observations antérieures (PËrTr, I972) nous avaient déjà montré que la différence dans les temps de pâturage entre des veaux recevant différentes quantités de lait s'amenuisait du printemps (à l'âge de 3-4 mois) à l'automne (7-8 mois). Il est possible que les quantités d'herbe ingérées soient souvent limitées à l'automne par la quantité et la qualité de l'herbe disponible. Dans la présente étude, les quantités d'herbe distribuées ont toujours permis I3,7 à Io,2 p. Ioo de refus, mais la proportion d'éléments morts et la teneur en cendres ont parfois été très importantes, au moins au cours de l'automne I973. HodGSON (I968) a par ailleurs montré l'importance de la digestibilité de l'herbe offerte sur les quantités de matière sèche ingérées par le veau. De plus, la distribution à l'auge n'a pas permis le tri important, que les veaux sont capables d'accomplir au pâturage (LEAVER, I974). Enfin, les variations de la teneur en matière sèche de l'herbe consécutives aux pluies d'automne ont également pu limiter les quantités ingérées, bien que ceci n'ait pas été clairement démontré dans le cas de jeunes veaux (Alder et CoOper, I967).

On peut remarquer par ailleurs que la quantité de matière sèche totale ingérée par jour semble liée à l'âge des veaux davantage qu'à leur poids ; cela pourrait résulter du développement du tube digestif qui peut être identique quelle que soit la vitesse de croissance des veaux. Ceux-ci consommeraient toujours le maximum d'herbe permis par les capacités de leurs réservoirs digestifs ; comme ils ont des poids différents, leur appétit par kilo de poids métabolique est donc plus élevé pour les veaux les plus légers. Les résultats des travaux en cours semblent confirmer cette hypothèse puisque nous avons observé que des veaux de même âge ayant $50 \mathrm{~kg}$ de poids vif d'écart, du fait des différences de quantités de lait bues, avaient cependant le même poids de tube digestif vide.

\section{III. - Bilan nutritionnel}

On a supposé qu'il n'existait aucune interaction d'ordre digestif entre le lait et l'herbe, le calcul de l'énergie apportée par chaque aliment étant fait indépendamment de l'énergie apportée par l'autre. La valeur en énergie brute du lait a été calculée à l'aide de la formule proposée par TYReLL et REID ( 1965$)$. En admettant que le taux butyreux et le taux de matières azotées du lait bu étaient identiques à ceux obtenus en I97I sur huit de ces vaches alors qu'elles étaient traites régulièrement, soit respectivement $39,9 \mathrm{~g} / \mathrm{kg}$ de lait et $34, \mathrm{I} / \mathrm{kg}$ de lait, la valeur en énergie brute du kilogramme de lait est égale à $754 \mathrm{kcal}$. Les coefficients de transformation de l'énergie brute en énergie digestible et en énergie métabolisable étant respectivement de 0,97 et 0,92 (VerRorei, communication personnelle). Les concentrations du lait en énergies digestible et métabolisable sont de 732 et $694 \mathrm{kcal} / \mathrm{kg}$ de lait.

L'énergie digestible de l'herbe a été calculée à partir de la composition chimique et de la digestibilité de cette herbe selon les équations suivantes (Demarquir.hy, résultats non publiés ): 
E.B. $=4450+16,26 \times a$.

E.B. : Énergie brute en cal./g de matière organique.

$a$ : Taux de matières azotées en p. roo de la matière organique.

C.U.D. de l'énergie $=0,958 \times$ C.U.D. de la matière organique - 0,2r.

C.U.D. : coefficient d'utilisation digestive apparent en p. Ioo.

Pour l'herbe, le coefficient de transformation de 1'énergie digestible en énergie métabolisable est supposé égal à 0,82 .

Les quantités d'énergie métabolisable totales ingérées augmentent régulièrement dans le temps, de $7787 \mathrm{kcal} / \mathrm{j}$ au cours de la première quinzaine de mesures à $14397 \mathrm{kcal} / \mathrm{j}$ au cours de la dernière quinzaine (fig. 3).

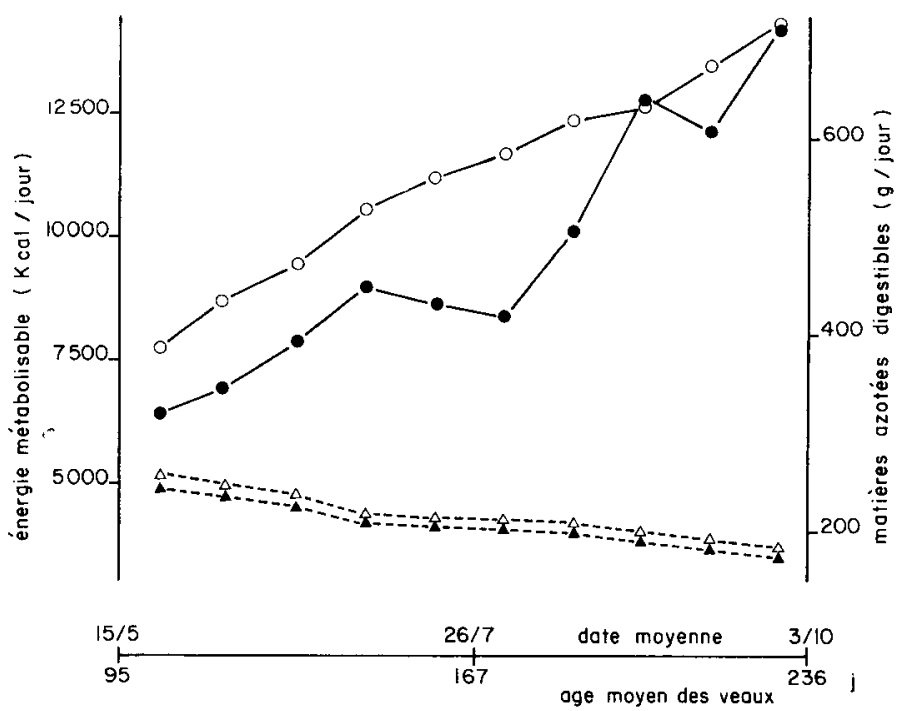

FiG. 3. - Quantitếs d'énergie métabolisable

et de matières azotées digestibles consommées par des veaux âgés de 3 à 8 mois

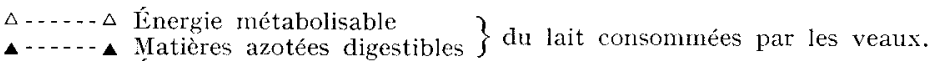

- - E Energie métabolisable $\{$ totales (lait + herbe) consommées par les veaux.

Les quantités de matières azotées digestibles totales ingérées ont augmenté de $320 \mathrm{~g}$ à $620 \mathrm{~g} / \mathrm{j}$ et par veau essentiellement du fait de l'augmentation des quantités d'herbe ingérées; cette augmentation est cependant moins régulière que celle de l'énergie du fait de la faible digestibilité de l'azote à certaines périodes, en particulier à la fin du $2^{\mathrm{e}}$ cycle de végétation en I972 (fig. I).

La différence de quantités d'herbe ingérées entre les lots $\mathrm{B}$ et $\mathrm{H}$ a permis de compenser 44,3 p. Ioo de la différence d'énergie métabolisable ingérée sous forme de lait entre les 2 lots au cours de la première période ( 3 à 5,5 mois), mais seulement II 6 p. Ioo au cours de la seconde période $(5,5$ à 8 mois) .

Nous avons étudié les variations d'énergie métabolisable totale ingérée (Y kcal) simultanément avec le poids métabolique $\left(\mathrm{X}=\mathrm{P}^{0,75} \mathrm{~kg}\right)$ et le gain de poids. L'introduction du gain de poids dans la régression n'améliore pas significativement la pré- 


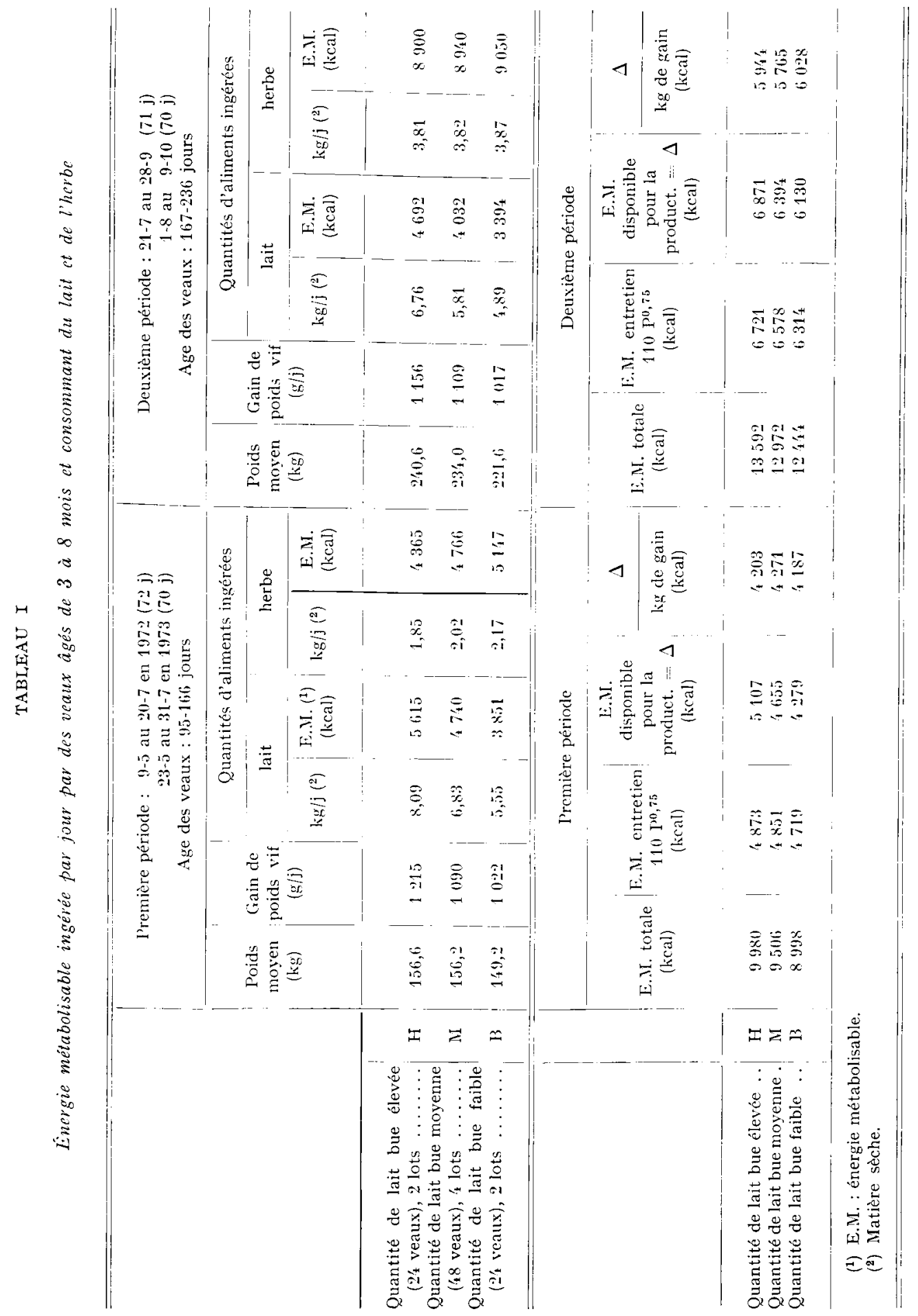


diction de $\mathrm{Y}$ du fait probablement de la faible variabilité de ce facteur et peut-être anssi de l'imprécision de l'estimation du gain de poids vif. L'énergie métabolisable totale ingérée $(\mathrm{Y})$ peut alors être estimée à partir du poids métabolique :

$$
\mathrm{Y}=2 \mathrm{I} 5 \mathrm{X}-\mathrm{I} 33 \text { avec } y^{2}=0,83 \quad \text { et } \quad \mathrm{S}_{y \cdot x}=30,4 \text {. }
$$

Si nous supposons que les besoins d'entretien en énergie métabolisable sont de I Io $\mathrm{kcal} / \mathrm{kg}$ de poids métabolique, les quantités d'énergie métabolisable disponibles par jour et pour la production sont pour les lots $\mathrm{H}$ et $\mathrm{B}$ respectivement de 5 Io $7 \mathrm{kcal}$ et $4279 \mathrm{kcal}$ pendant la première période, et de $687 \mathrm{I} \mathrm{kcal} \mathrm{et} 6 \mathrm{I} 30 \mathrm{kcal}$ pendant la denxième période. L'énergie métabolisable ramenée au $\mathrm{kg}$ de gain de poids vif est alors relativement constante pour la même période et égale en moyenne à $4233 \mathrm{kcal}$ pour la première période et à $5875 \mathrm{kcal}$ pour la deuxième période (tabl. $\mathrm{I}$ ).

Les différences entre les gains de poids vif journaliers des lots $\mathrm{H}$ et $\mathrm{B}$ sont de I93 à $139 \mathrm{~g}$ pendant la première et la deuxième période. Les différences entre les quantités d'énergie métabolisable disponibles pour la production sont de $828 \mathrm{kcal}$ et $74 \mathrm{I} \mathrm{kcal}$; ramenés au kilogramme de gain de poids vif, ces résultats sont voisins de ceux obtenus précédemment : 4290 kcal et 533 I kcal respectivement pour la première et la deuxième période.

L'énergie métabolisable nécessaire pour produire un kilogramme de gain de poids vif augmente donc de façon importante d'une période à l'autre ; cette différence est peut-être liée à une réduction de l'efficacité de l'énergie métabolisable due à la diminution de la part de la production laitière dans la ration. Cependant, cette évolution n'explique certainement pas complètement le phénomène ; la valeur calorifique du kilogramme de croît, donc les besoins de production, doivent également augmenter avec les poids des veaux. C'est pourquoi, nous avons relié la quantité d'énergie métabolisable pour la production et par kilogramme de gain de poids vif (E kcal) au poids vif $(\mathrm{P} \mathrm{kg})$ par l'équation $\mathrm{I}_{i}=\alpha \mathrm{P}^{\beta}$, sachant que l'énergie fixée par kilogramme de gain de poids varie selon une relation similaire (REID ct al., I968).

Nous avons estimé les coefficients $\alpha$ et $\beta$ à partir d'une droite de régression logarithmique :

$$
\log (\mathrm{I} ; \mathrm{kcal})=4, \mathbf{I} 6+0,83 \log (\mathrm{P} \mathrm{kg}) \quad r^{2}=0,49
$$

$\mathrm{Si}$ on admet une valeur moyenne pour les besoins d'entretien de IIo $\mathrm{kcal} / \mathrm{kg}$ de poids métabolique, il est possible d'obtenir les besoins en énergie métabolisable totale (E.M.T. kcal) à partir du poids $(\mathrm{P} \mathrm{kg}$ ) et du gain de poids vif journalier (G.M.Q. kg).

$$
\text { F.M.T. }=\text { I Io } \mathrm{P}^{0,75}+64,3 \mathrm{P}^{0,83} \cdot(\mathrm{G} . \mathrm{M} . \mathrm{Q} .)
$$

\section{CONCLUSION}

Les quantités de matières sèches d'herbe ingérées par un veau élevé au pis augmentent considérablement avec l'âge. Elles sont d'autant plus importantes qu'il a moins de lait à sa disposition; la compensation du manque de lait par de l'herbe a été pour l'énergie métabolisable dans notre expérimentation de 44,3 p. Ioo entre 3 
et 5,5 mois et de I I, 6 p. Ioo entre 5,5 et $\delta$ mois. Elle n'est cependant pas complète car le veau n'arrive pas à ingérer suffisamment d'herbe, sans doute du fait d'une limite de sa capacité physique d’ingestion liée au développement de son tube digestif. I) ce fait, la quantité de lait disponib!e reste un facteur limitant la croissance du veau jusqu'au sevrage. Ces résultats ont été obtenus avec un seul type de fourrage : du ray-grass anglais dont la qualité évolue avec la saison; il importerait de mieux connaître l'influence de la qualité des fourrages sur leur ingestibilité par ce type de veau et leurs possibilités de substitution au lait.

Avec $6,3 \mathrm{~kg}$ de lait par jour et de l'herbe de bonne qualité distribuée à volonté, les veaux ont eu une croissance journalière moyenne de I I $66 \mathrm{~g}$ sans apport d'aliment concentré. Un accroissement de la quantité de lait bue de I $\mathrm{kg}$ a permis une augmentation de la croissance journalière des veaux de $65 \mathrm{~g}$ de l'âge de 3 mois jusqu'au sevrage. L'herbe consommée a représenté $69 \mathrm{p}$. Ioo de l'énergie métabolisable totale consommée pendant la deuxième partie de l'expérimentation; elle doit donc être de très bonne qualité.

L'augmentation considérable des quantités d'herbe ingérées par les veaux entraîne pour le troupeau de vaches allaitantes des besoins totaux (vaches + veaux) croissants au cours de la saison ou à peu près constants si on tient compte de la reconstitution des réserves corporelles des vaches au printemps. Ionc, si on veut maintenir à l'automne une croissance élevée des veaux et un état satisfaisant de leurs mères, les surfaces qui leur sont allouées doivent être alors accrues de façon importante.

Resu pour publication en août 19\%6.

\title{
SUMMARY
}

GRASS AND MILK INTAKE LEVEIS IN SUCKIED CALVES

\begin{abstract}
A total number of 96 purebred Nomman or crossbred Charolais $\times$ Noman calves were reared indoors on two consccutive ycars from 3 till 8 months of age. Twice a day, they were nursed by Norman cows (3 calves per cow). Milk production of cach cow was estimated every two wecks by weighing its 3 calves before and after sucking. Each ycar, 4 groups of 2 calves sucking different amounts of milk, were fed ad libitum cut green ray-grass. Grass intake was measured each week on 5 consecutive days. Ijgestibility of the grass was estimated on sheep cluring 9 weeks of the first year and 20 weeks of the second year.

Average live weight of calves increased from i 5 to 27 I $\mathrm{kg}$ (9.5 th to 236 th lay of age) (fig. I c). ()ne $\mathrm{kg}$ of excess daily milk intake increased daily live weight gain by $6.5 \mathrm{~g}$ as well from 3 to 5.5 months (first period) as from 5.5 to 8 months (second period). During the experimental period, daily dry matter intake of grass considerably increased from $\mathrm{I}, \mathrm{I} 2$ to,$+ 76 \mathrm{~kg}$ per calf (fig. I b). In addition, the dry matter intake of grass per kilogramme of metabolic weight --P0,75 — increased with decreasing amount of milk available between calves.

Daily intake of metabolisable energy and digestible crude protein per calf almost doubled during the observations. The higher intake of grass by the calves sucking less milk did not compensate for this lack of milk. On a metabolisable energy basis, compensation was $+4,3$ p. Ioo and I .6 p. 100 from 3 to 5,5 months and frem 5,5, to 8 months respectively. Estimated metabolisable energy available for $\mathrm{I} \mathrm{kg}$ of live weight gain (total energy minus energy for maintenance) was the same for all groups of calves and increased from +23.3 kcal during the first period to 5876 kcal during the second period
\end{abstract}




\section{RÉFÉRENCFS BIBLIOGRAPHIQUES}

Ardir F. E., r970. Comparative studies of perennial ryegrass, Timothy and neadow Foscue; 2-results obtained in Grazing experiments. J. Br. Grassld Soc., 25, 53-64.

Alder F. E., Coopre E. M., r 967 . Comparative studies of perennial ryegrass and cooksfoot as food for the calf. J. Agric. Sci., 68, 33 I-346.

Brvmby P. J., Walker D. K., Gallagher R. M., I963. I'actors associated with growth in beef cattle. N.Z. J. Agric. Res., 6, 526-537.

Demarguidy C., Weiss P., r970. Tableaux de la valeur alimentaire des fourrages. I.N.R.A.-S.E.I., Eitude $n^{\circ} 42$.

Furr R. D., Nusson A. B., I964. Effect of level of supplemental winter fecd on calf weight and on milk production of fall calving range beef cows. J. Anim. Sci., 23, 775-78I.

Gienson 1'. A., 1971. Grass as a food for calves. Ir. J. Agric. Re's., 10, 15I-159.

Hongsox J., I 968 . The relationship between the digestibility of a sward and the herbage consumption of grazing calves. J. Agric. Sici, $70,47-5 \mathrm{I}$.

Kaiser A. (., 1973. Pre-weaning level of milk and reticulo-rumen development in the grazing calf. II I. World conforence of Animal Production, vol. 1, $5(d)$, I6.

I.BAVER J. D., 1974. Rearing of dairy cattle. V. The effect of stocking rate on animal and herbage production on a grazing systen for calves and heifers. Anim. Prod., 18, 273-284.

Je Neñore P', P'etit M., Mubler A., I976a. P'roduction laitière de vaches Normandés à la traite oll à l'allaitement. Ann. Zootech., 25, 533542 .

Ll: Neindre l'., Petre M., Tomassone R., Rovx C., 1976 b. Production laitière de vaches allaitantes et croistance de leurs veaux. I. Race Limousine. Ann. Zootech, 25, 22 I-24I.

Marmev C.-M., ig6r. F́tude du développenent du tractus direstif. Ann. Nutr. et Alim., 14, $263-266$.

Mather C.-M., Wegat-Litrf: E., rg6r. Mise au point d'une méthode d'alinentation des veaux d'élevage. I. Détermination de la quantité de lait nécessaire. Ann. Zootech., 10, 161-I75.

Mritox A. A., RigGs J. K., Nlosox L. A., CAktwright J. C., ig67. Milk production, composition and calf gains of Angus, Charolais and Hcreford cows. J. Anim. Sci, 26, 804-809.

Nivilif W. E., Jr, I962. Infuence of dam's milk production and other factors on r2o and 240 day weight of hereford calves. J. Anim. Sci., 21, 315-320.

P'iт M., I972. Enmploi du temps des troupeaux de vaches-mères et de leurs veaux sur les pâturages d'altitude de l'Aubrac. Ann. Zootech., 21, 5-27.

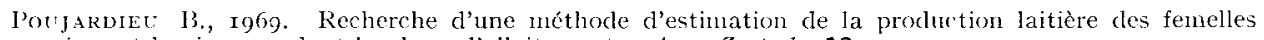
ovines et bovines pendant lat phase d'allaitement. Ann. Zootch., 18, 299-315.

Rieid J. T., Brinsadoun A., Bull L. S., Jurton J. H., Gileeson 1'. A., Han I. K., Joo Y. D.,

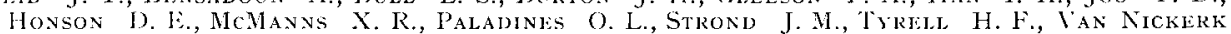
13. O. H., Millington G. M., I968. Some pecularities in the body composition of animals. In body composition in animals and man. Washington, National Academy of Science.

TYRLLL H. I., Rlin J. T. J., I965. Fredirtion of the encrgy value of cow's milk. J. Iair. Sci, 48, I $215-1223$. 